\title{
Multiple monotone positive solutions for higher order differential equations with integral boundary conditions
}

Xinan $\mathrm{Hao}^{*}$ and Lishan Liu

"Correspondence:

haoxinan2004@163.com School of Mathematical Sciences,

Qufu Normal University, Qufu,

Shandong, 273165, P.R. China

\section{Springer}

\begin{abstract}
This paper investigates the higher order differential equations with nonlocal boundary conditions$$
\left\{\begin{array}{l}
u^{(n)}(t)+f\left(t, u(t), u^{\prime}(t), \ldots, u^{(n-2)}(t)\right)=0, \quad t \in(0,1) \\
u(0)=u^{\prime}(0)=\cdots=u^{(n-3)}(0)=0, \\
u^{(n-2)}(0)=\int_{0}^{1} u^{(n-2)}(s) d A(s), \quad u^{(n-2)}(1)=\int_{0}^{1} u^{(n-2)}(s) d B(s) .
\end{array}\right.
$$

The existence results of multiple monotone positive solutions are obtained by means of fixed point index theory for operators in a cone.
\end{abstract}

MSC: 34B10; 34B18

Keywords: monotone positive solutions; multiplicity; higher order differential equations; nonlocal boundary conditions

\section{Introduction}

In this paper, we are concerned with the existence of multiple monotone positive solutions for the higher order differential equation

$$
u^{(n)}(t)+f\left(t, u(t), u^{\prime}(t), \ldots, u^{(n-2)}(t)\right)=0, \quad t \in(0,1)
$$

subject to the following integral boundary conditions:

$$
\left\{\begin{array}{l}
u(0)=u^{\prime}(0)=\cdots=u^{(n-3)}(0)=0, \\
u^{(n-2)}(0)=\int_{0}^{1} u^{(n-2)}(s) d A(s), \quad u^{(n-2)}(1)=\int_{0}^{1} u^{(n-2)}(s) d B(s),
\end{array}\right.
$$

where $n \geq 3, f:[0,1] \times\left(\mathbf{R}^{+}\right)^{n-1} \rightarrow \mathbf{R}^{+}$is continuous in which $\mathbf{R}^{+}=[0,+\infty), A$ and $B$ are right continuous on $[0,1)$, left continuous at $t=1$, and nondecreasing on $[0,1]$, with $A(0)=$ $B(0)=0 ; \int_{0}^{1} v(s) d A(s)$ and $\int_{0}^{1} v(s) d B(s)$ denote the Riemann-Stieltjes integrals of $v$ with respect to $A$ and $B$, respectively.

Boundary value problems (BVPs for short) for nonlinear differential equations arise in many areas of applied mathematics and physics. Many authors have discussed the existence of positive solutions for second order or higher order differential equations with 
boundary conditions defined at a finite number of points, for instance, [1-12]. In [2], Graef and Yang considered the following $n$ th-order multi-point BVP:

$$
\left\{\begin{array}{l}
u^{(n)}(t)+\lambda g(t) f(u(t))=0, \quad t \in(0,1), \\
u(0)=u^{\prime}(0)=\cdots=u^{(n-2)}(0)=\sum_{i=1}^{m} a_{i} u^{(n-2)}\left(\xi_{i}\right)-u^{(n-2)}(1)=0
\end{array}\right.
$$

where $n \geq 3, \lambda>0$ is a parameter, $g$ and $f$ are continuous functions, $\frac{1}{2} \leq \xi_{1}<\xi_{2}<\cdots<\xi_{m}<$ $1, a_{i}>0$ for $1 \leq i \leq m$ and $\sum_{i=1}^{m} a_{i}=1$. The authors obtained the existence and nonexistence results of positive solutions by using Krasnosel'skii's fixed point theorem in cones. In [5], we studied the following second order $m$-point nonhomogeneous BVP:

$$
\left\{\begin{array}{l}
u^{\prime \prime}(t)+a(t) f(t, u)=0, \quad t \in(0,1) \\
u(0)=0, u(1)-\sum_{i=1}^{m-2} k_{i} u\left(\xi_{i}\right)=b
\end{array}\right.
$$

where $b>0, k_{i}>0(i=1,2, \ldots, m-2), 0<\xi_{1}<\xi_{2}<\cdots<\xi_{m-2}<1, \sum_{i=1}^{m-2} k_{i} \xi_{i}<1$. The authors obtained the existence, nonexistence and multiplicity of positive solutions by using the Krasnosel'skii-Guo fixed point theorem, the upper-lower solutions method and topological degree theory.

Boundary value problems with integral boundary conditions for ordinary differential equations represent a very interesting and important class of problems and arise in the study of various physical, biological and chemical processes [13-15], such as heat conduction, chemical engineering, underground water flow, thermo-elasticity, and plasma physics. They include two, three, multi-point and nonlocal BVPs as special cases. The existence and multiplicity of positive solutions for such problems have received a great deal of attention, see [16-32] and the references therein. In [17], Feng, Ji and Ge considered the existence and multiplicity of positive solutions for a class of nonlinear boundary value problems of second order differential equations with integral boundary conditions in ordered Banach spaces

$$
\left\{\begin{array}{l}
x^{\prime \prime}(t)+f(t, x(t))=\theta, \quad t \in(0,1), \\
x(0)=\int_{0}^{1} g(t) x(t) d t, \quad x(1)=\theta, \quad \text { or } \\
x(0)=\theta, \quad x(1)=\int_{0}^{1} g(t) x(t) d t .
\end{array}\right.
$$

The arguments are based upon a specially constructed cone and fixed point theory in a cone for strict set contraction operators.

Motivated by the works mentioned above, in this paper, we consider the existence of multiple monotone positive solutions for BVP (1.1) and (1.2). In comparison with previous works, our paper has several new features. Firstly, we consider higher order boundary value problems, and we allow the nonlinearity $f$ to contain derivatives of the unknown function $u(t)$ up to $n-2$ order. Secondly, we discuss the boundary value problem with integral boundary conditions, i.e., BVP (1.1) and (1.2), which includes two-point, threepoint, multi-point and nonlocal boundary value problems as special cases. Thirdly, we consider the existence of multiple monotone positive solutions. To our knowledge, few papers have considered the monotone positive solutions for a higher order differential equation with integral boundary conditions. We shall emphasize here that with these new 
features our work improves and generalizes the results of [2] and some other known results to some degree. In this work we shall also utilize the following fixed point theorem in cones.

Lemma 1.1 ([33,34]) Let $K$ be a cone in a Banach space E. Let $D$ be an open bounded subset of $E$ with $D_{K}=D \cap K \neq \emptyset$ and $\bar{D}_{K} \neq K$. Assume that $A: \bar{D}_{K} \rightarrow K$ is a compact operator such that $u \neq A u$ for $u \in \partial D_{K}$. Then the following results hold.

(1) If $\|A u\| \leq\|u\|, u \in \partial D_{K}$, then $i_{K}\left(A, D_{K}\right)=1$.

(2) If there exists $e \in K \backslash\{0\}$ such that $u \neq A u+\lambda$ e for all $u \in \partial D_{K}$ and $\lambda>0$, then $i_{K}\left(A, D_{K}\right)=0$.

(3) Let $U$ be open in $E$ such that $\bar{U} \subset D_{K}$. If $i_{K}\left(A, D_{K}\right)=1$ and $i_{K}\left(A, U_{K}\right)=0$, then $A$ has a fixed point in $D_{K} \backslash \bar{U}_{K}$. The same result holds if $i_{K}\left(A, D_{K}\right)=0$ and $i_{K}\left(A, U_{K}\right)=1$.

\section{Preliminary lemmas}

Let $E=\left\{u \in C^{n-2}[0,1]: u^{(i)}(0)=0,0 \leq i \leq n-3\right\}$, then $E$ is a Banach space with the norm $\|u\|=\sup _{t \in[0,1]}\left|u^{(n-2)}(t)\right|$ for each $u \in E$.

We make the following assumptions:

$\left(H_{1}\right) f:[0,1] \times\left(\mathbf{R}^{+}\right)^{n-1} \rightarrow \mathbf{R}^{+}$is continuous.

$\left(H_{2}\right) k_{1}>0, k_{4}>0, k>0$, where $k=k_{1} k_{4}-k_{2} k_{3}$,

$$
\begin{aligned}
& k_{1}=1-\int_{0}^{1}(1-s) d A(s), \quad k_{2}=\int_{0}^{1} s d A(s), \\
& k_{3}=\int_{0}^{1}(1-s) d B(s), \quad k_{4}=1-\int_{0}^{1} s d B(s) .
\end{aligned}
$$

Lemma 2.1 Assume that $\left(H_{2}\right)$ holds. Then, for any $y \in C[0,1]$, the BVP

$$
\left\{\begin{array}{l}
-u^{\prime \prime}(t)=y(t), \quad t \in(0,1), \\
u(0)=\int_{0}^{1} u(t) d A(t), \quad u(1)=\int_{0}^{1} u(t) d B(t)
\end{array}\right.
$$

has a unique solution $u$ that can be expressed in the form

$$
u(t)=\int_{0}^{1} H(t, s) y(s) d s, \quad t \in[0,1]
$$

where

$$
\begin{aligned}
& H(t, s)=G(t, s)+\frac{t k_{3}+(1-t) k_{4}}{k} \mathcal{G}_{A}(s)+\frac{t k_{1}+(1-t) k_{2}}{k} \mathcal{G}_{B}(s), \\
& \mathcal{G}_{A}(s)=\int_{0}^{1} G(\tau, s) d A(\tau), \quad \mathcal{G}_{B}(s)=\int_{0}^{1} G(\tau, s) d B(\tau), \\
& G(t, s)= \begin{cases}t(1-s), & 0 \leq t \leq s \leq 1, \\
s(1-t), & 0 \leq s \leq t \leq 1 .\end{cases}
\end{aligned}
$$


Proof Firstly, we prove that if $u$ is a solution of BVP (2.1), then it will take the form of (2.2). Now, integrating differential equation (2.1) from 0 to $t$ twice, we have

$$
u(t)=u(0)+u^{\prime}(0) t-\int_{0}^{t}(t-s) y(s) d s .
$$

Letting $t=1$ in (2.4), we get

$$
u^{\prime}(0)=u(1)-u(0)+\int_{0}^{1}(1-s) y(s) d s
$$

Substituting the boundary conditions of (2.1) and (2.5) into (2.4) yields

$$
\begin{aligned}
u(t) & =u(0)+\left[u(1)-u(0)+\int_{0}^{1}(1-s) y(s) d s\right] t-\int_{0}^{t}(t-s) y(s) d s \\
& =(1-t) \int_{0}^{1} u(t) d A(t)+t \int_{0}^{1} u(t) d B(t)+\int_{0}^{1} G(t, s) y(s) d s,
\end{aligned}
$$

and, consequently,

$$
\begin{aligned}
\int_{0}^{1} u(t) d A(t)= & \int_{0}^{1}(1-t) d A(t) \int_{0}^{1} u(t) d A(t)+\int_{0}^{1} t d A(t) \int_{0}^{1} u(t) d B(t) \\
& +\int_{0}^{1} \int_{0}^{1} G(t, s) y(s) d s d A(t), \\
\int_{0}^{1} u(t) d B(t)= & \int_{0}^{1}(1-t) d B(t) \int_{0}^{1} u(t) d A(t)+\int_{0}^{1} t d B(t) \int_{0}^{1} u(t) d B(t) \\
& +\int_{0}^{1} \int_{0}^{1} G(t, s) y(s) d s d B(t) .
\end{aligned}
$$

Solving the above two equations for $\int_{0}^{1} u(t) d A(t)$ and $\int_{0}^{1} u(t) d B(t)$, we have

$$
\left(\begin{array}{cc}
k_{1} & -k_{2} \\
-k_{3} & k_{4}
\end{array}\right)\left(\begin{array}{l}
\int_{0}^{1} u(t) d A(t) \\
\int_{0}^{1} u(t) d B(t)
\end{array}\right)=\left(\begin{array}{c}
\int_{0}^{1} \int_{0}^{1} G(t, s) y(s) d s d A(t) \\
\int_{0}^{1} \int_{0}^{1} G(t, s) y(s) d s d B(t)
\end{array}\right)
$$

and so

$$
\left(\begin{array}{l}
\int_{0}^{1} u(t) d A(t) \\
\int_{0}^{1} u(t) d B(t)
\end{array}\right)=k^{-1}\left(\begin{array}{ll}
k_{4} & k_{2} \\
k_{3} & k_{1}
\end{array}\right)\left(\begin{array}{l}
\int_{0}^{1} \int_{0}^{1} G(t, s) y(s) d s d A(t) \\
\int_{0}^{1} \int_{0}^{1} G(t, s) y(s) d s d B(t)
\end{array}\right) .
$$

Hence, (2.2) follows from (2.6) and (2.7).

Next we prove that the $u$ given by (2.2) satisfies the differential equation and boundary conditions of (2.1). From (2.2), we have

$$
\begin{aligned}
u(t)= & \int_{0}^{t} s(1-t) y(s) d s+\int_{t}^{1} t(1-s) y(s) d s \\
& +\frac{t k_{3}+(1-t) k_{4}}{k} \int_{0}^{1}\left[\int_{0}^{1} G(\tau, s) d A(\tau)\right] y(s) d s \\
& +\frac{t k_{1}+(1-t) k_{2}}{k} \int_{0}^{1}\left[\int_{0}^{1} G(\tau, s) d B(\tau)\right] y(s) d s .
\end{aligned}
$$


Direct differentiation of (2.8) gives $u^{\prime \prime}(t)=-y(t)$. Also, from (2.2) we have

$$
\begin{aligned}
\int_{0}^{1} u(t) d A(t)= & \int_{0}^{1} \int_{0}^{1} G(t, s) y(s) d s d A(t) \\
& +k^{-1}\left[k_{3} k_{2}+k_{4}\left(1-k_{1}\right)\right] \int_{0}^{1}\left[\int_{0}^{1} G(\tau, s) d A(\tau)\right] y(s) d s \\
& +k^{-1}\left[k_{1} k_{2}+k_{2}\left(1-k_{1}\right)\right] \int_{0}^{1}\left[\int_{0}^{1} G(\tau, s) d B(\tau)\right] y(s) d s \\
= & k^{-1} k_{4} \int_{0}^{1} \int_{0}^{1} G(\tau, s) y(s) d A(\tau) d s \\
& +k^{-1} k_{2} \int_{0}^{1} \int_{0}^{1} G(\tau, s) y(s) d B(\tau) d s
\end{aligned}
$$

and, similarly,

$$
\int_{0}^{1} u(t) d B(t)=k^{-1} k_{3} \int_{0}^{1} \int_{0}^{1} G(\tau, s) y(s) d A(\tau) d s+k^{-1} k_{1} \int_{0}^{1} \int_{0}^{1} G(\tau, s) y(s) d B(\tau) d s .
$$

Therefore, by solving the above two equations with the double integrals as unknowns, we have

$$
\int_{0}^{1} \int_{0}^{1} G(\tau, s) y(s) d A(\tau) d s=k_{1} \int_{0}^{1} u(t) d A(t)-k_{2} \int_{0}^{1} u(t) d B(t)
$$

and

$$
\int_{0}^{1} \int_{0}^{1} G(\tau, s) y(s) d B(\tau) d s=-k_{3} \int_{0}^{1} u(t) d A(t)+k_{4} \int_{0}^{1} u(t) d B(t) .
$$

Hence (2.6) follows from (2.2), (2.9) and (2.10), and thus $u(0)=\int_{0}^{1} u(t) d A(t), u(1)=$ $\int_{0}^{1} u(t) d B(t)$. This completes the proof.

Defining

$$
G_{3}(t, s)=\int_{0}^{t} H(v, s) d v, \quad G_{i}(t, s)=\int_{0}^{t} G_{i-1}(v, s) d v, \quad i \geq 4,
$$

then $G_{n}(t, s)$ is the Green function of BVP (1.1) and (1.2). Moreover, solving BVP (1.1) and (1.2) is equivalent to finding a solution of the following integral equation:

$$
u(t)=\int_{0}^{1} G_{n}(t, s) f\left(s, u(s), u^{\prime}(s), \ldots, u^{(n-2)}(s)\right) d s, \quad t \in[0,1] .
$$

Remark 2.1 If $\left(H_{2}\right)$ holds, then for any $t, s \in[0,1]$, it is easy to testify that

$$
G(t, s) \geq 0, \quad H(t, s) \geq 0, \quad G_{n}(t, s) \geq 0, \quad n \geq 3 .
$$

Lemma 2.2 Let $\delta \in\left(0, \frac{1}{2}\right)$, then for any $t \in[\delta, 1-\delta], \eta, s \in[0,1]$, we have

$$
H(t, s) \geq \delta H(\eta, s) .
$$


Proof It is easy to show that $G(t, s) \geq \delta G(\eta, s), \forall t \in[\delta, 1-\delta], \eta, s \in[0,1]$. For $t \in[\delta, 1-\delta]$, $\eta, s \in[0,1]$, we have

$$
\begin{aligned}
H(t, s)= & G(t, s)+\frac{t k_{3}+(1-t) k_{4}}{k} \int_{0}^{1} G(\tau, s) d A(\tau)+\frac{t k_{1}+(1-t) k_{2}}{k} \int_{0}^{1} G(\tau, s) d B(\tau) \\
\geq & \delta G(\eta, s)+\frac{\delta\left(k_{3}+k_{4}\right)}{k} \int_{0}^{1} G(\tau, s) d A(\tau)+\frac{\delta\left(k_{1}+k_{2}\right)}{k} \int_{0}^{1} G(\tau, s) d B(\tau) \\
\geq & \delta\left[G(\eta, s)+\frac{\eta k_{3}+(1-\eta) k_{4}}{k} \int_{0}^{1} G(\tau, s) d A(\tau)\right. \\
& \left.+\frac{\eta k_{1}+(1-\eta) k_{2}}{k} \int_{0}^{1} G(\tau, s) d B(\tau)\right] \\
= & \delta H(\eta, s) .
\end{aligned}
$$

For any $s \in[0,1]$, we define $\mathcal{H}(s)=\max _{t \in[0,1]} H(t, s)$. From Lemma 2.2 , we know that

$$
\delta \mathcal{H}(s) \leq H(t, s) \leq \mathcal{H}(s), \quad t \in[\delta, 1-\delta], s \in[0,1]
$$

Lemma 2.3 Assume that $\left(H_{2}\right)$ holds. If $u \in C^{n}[0,1]$ satisfies the boundary conditions (1.2) and

$$
u^{(n)}(t) \leq 0, \quad t \in[0,1]
$$

then

$$
u(t) \geq 0 \quad \text { and } \quad u^{\prime}(t) \geq 0 \quad \text { for } t \in[0,1]
$$

Proof Let $m(t)=u^{(n-2)}(t), t \in[0,1]$, then we have

$$
\left\{\begin{array}{l}
m^{\prime \prime}(t) \leq 0, \quad t \in[0,1], \\
m(0)=\int_{0}^{1} m(t) d A(t), \quad m(1)=\int_{0}^{1} m(t) d B(t) .
\end{array}\right.
$$

For $t \in[0,1], m^{\prime \prime}(t) \leq 0$ implies that

$$
m(t)=m[(1-t) \cdot 0+t \cdot 1] \geq(1-t) m(0)+t m(1)
$$

Thus

$$
\begin{aligned}
m(0) & =\int_{0}^{1} m(t) d A(t) \geq m(0) \int_{0}^{1}(1-t) d A(t)+m(1) \int_{0}^{1} t d A(t) \\
& =\left(1-k_{1}\right) m(0)+k_{2} m(1)
\end{aligned}
$$

i.e.,

$$
m(0) \geq \frac{k_{2}}{k_{1}} m(1)
$$


On the other hand,

$$
\begin{aligned}
m(1) & =\int_{0}^{1} m(t) d B(t) \geq m(0) \int_{0}^{1}(1-t) d B(t)+m(1) \int_{0}^{1} t d B(t) \\
& =k_{3} m(0)+\left(1-k_{4}\right) m(1),
\end{aligned}
$$

and so

$$
m(1) \geq \frac{k_{3}}{k_{4}} m(0) \geq \frac{k_{2} k_{3}}{k_{1} k_{4}} m(1)
$$

i.e., $k m(1) \geq 0$, therefore $m(1) \geq 0$, and so $m(0) \geq 0$.

Now, $m(0) \geq 0, m(1) \geq 0$ and $m(t)$ is concave downward, so we have

$$
m(t)=u^{(n-2)}(t) \geq 0, \quad t \in[0,1] .
$$

From (2.14) and $u(0)=u^{\prime}(0)=\cdots=u^{(n-3)}(0)=0$, we obtain (2.13). This completes the proof of Lemma 2.3.

Remark 2.2 From Lemma 2.3, if $u$ is a positive solution of BVP (1.1) and (1.2), then $u$ is nondecreasing on $[0,1]$, i.e., $u$ is a monotone positive solution of BVP (1.1) and (1.2).

Let

$$
K=\left\{u \in E: u^{(n-2)}(t) \geq 0, t \in[0,1], u^{(n-2)}(t) \geq \delta\|u\|, t \in[\delta, 1-\delta]\right\} .
$$

Obviously, $K$ is a cone in $E$. For any $\rho>0$, let $K_{\rho}=\{u \in K:\|u\|<\rho\}, \partial K_{\rho}=\{u \in K:\|u\|=$ $\rho\}$ and $\bar{K}_{\rho}=\{u \in K:\|u\| \leq \rho\}$. Define an operator $T: K \rightarrow E$ as follows:

$$
(T u)(t)=\int_{0}^{1} G_{n}(t, s) f\left(s, u(s), u^{\prime}(s), \ldots, u^{(n-2)}(s)\right) d s, \quad t \in[0,1] .
$$

Then $u$ is a solution of BVP (1.1) and (1.2) if and only if $u$ solves the operator equation $u=T u$.

Lemma 2.4 Suppose that $\left(H_{1}\right)$ and $\left(H_{2}\right)$ hold, then $T: K \rightarrow K$ is completely continuous.

Proof For all $u \in K, t \in[0,1]$, by $\left(H_{1}\right),(2.11),(2.12)$ and (2.15), we have

$$
\begin{aligned}
(T u)^{(n-2)}(t) & =\int_{0}^{1} H(t, s) f\left(s, u(s), u^{\prime}(s), \ldots, u^{(n-2)}(s)\right) d s \geq 0, \\
(T u)^{(n-2)}(t) & =\int_{0}^{1} H(t, s) f\left(s, u(s), u^{\prime}(s), \ldots, u^{(n-2)}(s)\right) d s \\
& \leq \int_{0}^{1} \mathcal{H}(s) f\left(s, u(s), u^{\prime}(s), \ldots, u^{(n-2)}(s)\right) d s,
\end{aligned}
$$

and

$$
\|T u\| \leq \int_{0}^{1} \mathcal{H}(s) f\left(s, u(s), u^{\prime}(s), \ldots, u^{(n-2)}(s)\right) d s
$$


Thus, further from the first inequality of (2.12), we have

$$
\begin{aligned}
(T u)^{(n-2)}(t) & =\int_{0}^{1} H(t, s) f\left(s, u(s), u^{\prime}(s), \ldots, u^{(n-2)}(s)\right) d s \\
& \geq \delta \int_{0}^{1} \mathcal{H}(s) f\left(s, u(s), u^{\prime}(s), \ldots, u^{(n-2)}(s)\right) d s \\
& \geq \delta\|T u\|, \quad t \in[\delta, 1-\delta] .
\end{aligned}
$$

Hence, $T u \in K$ and $T(K) \subset K$.

Next by standard methods and the Ascoli-Arzela theorem, one can prove that $T: K \rightarrow K$ is completely continuous. So this is omitted.

Let

$$
\begin{aligned}
\Omega_{\rho} & =\left\{u \in K: \min _{\delta \leq t \leq 1-\delta} u^{(n-2)}(t)<\delta \rho\right\} \\
& =\left\{u \in E: u^{(n-2)}(t) \geq 0, t \in[0,1], \delta\|u\| \leq \min _{\delta \leq t \leq 1-\delta} u^{(n-2)}(t)<\delta \rho\right\} .
\end{aligned}
$$

Proceeding as for the proof of Lemma 2.5 in [33], we have the following.

Lemma $2.5 \Omega_{\rho}$ has the following properties:

(a) $\Omega_{\rho}$ is open relative to $K$;

(b) $K_{\delta \rho} \subset \Omega_{\rho} \subset K_{\rho}$;

(c) $u \in \partial \Omega_{\rho}$ if and only if $\min _{\delta \leq t \leq 1-\delta} u^{(n-2)}(t)=\delta \rho$;

(d) if $u \in \partial \Omega_{\rho}$, then $\delta \rho \leq u^{(n-2)}(t) \leq \rho$ for $\delta \leq t \leq 1-\delta$.

Now for convenience we introduce the following notations:

$$
\begin{aligned}
& f_{\delta \rho, \rho}=\min \left\{\min _{\delta \leq t \leq 1-\delta} \frac{f\left(t, u_{0}, \ldots, u_{n-2}\right)}{\rho}: u_{0}, u_{1}, \ldots, u_{n-3} \in[0, \rho], u_{n-2} \in[\delta \rho, \rho]\right\} \\
& f^{0, \rho}=\max \left\{\max _{0 \leq t \leq 1} \frac{f\left(t, u_{0}, u_{1}, \ldots, u_{n-2}\right)}{\rho}:\left(u_{0}, u_{1}, \ldots, u_{n-2}\right) \in[0, \rho]^{n-1}\right\} \\
& f^{0}=\lim _{u_{0}, u_{1}, \ldots, u_{n-2} \rightarrow 0} \max _{0 \leq t \leq 1} \frac{f\left(t, u_{0}, u_{1}, \ldots, u_{n-2}\right)}{u_{n-2}}, \\
& f^{\infty}=\lim _{u_{0}+u_{1}+\cdots+u_{n-2} \rightarrow+\infty} \max _{0 \leq t \leq 1} \frac{f\left(t, u_{0}, u_{1}, \ldots, u_{n-2}\right)}{u_{0}+u_{1}+\cdots+u_{n-2}}, \\
& f_{0}=\lim _{u_{0}, u_{1}, \ldots, u_{n-2} \rightarrow 0} \min _{\delta \leq t \leq 1-\delta} \frac{f\left(t, u_{0}, u_{1}, \ldots, u_{n-2}\right)}{u_{n-2}}, \\
& f_{\infty}=\lim _{u_{0}+u_{1}+\cdots+u_{n-2} \rightarrow+\infty \delta \leq t \leq 1-\delta} \frac{f\left(t, u_{0}, u_{1}, \ldots, u_{n-2}\right)}{u_{0}+u_{1}+\cdots+u_{n-2}}, \\
& m=\left(\max _{0 \leq t \leq 1} \int_{0}^{1} H(t, s) d s\right)^{-1}, \quad M=\left(\min _{\delta \leq t \leq 1-\delta} \int_{\delta}^{1-\delta} H(t, s) d s\right)^{-1} .
\end{aligned}
$$

To prove our main results, we need the following lemmas. 
Lemma 2.6 Assume that $\left(H_{1}\right),\left(H_{2}\right)$ hold and $f$ satisfies

$$
f^{0, \rho} \leq m \text { and } u \neq T u \quad \text { for } u \in \partial K_{\rho}
$$

then $i_{K}\left(T, K_{\rho}\right)=1$.

Proof For $u \in \partial K_{\rho}$, we have $0 \leq u^{(n-2)}(t) \leq \rho$ and $0 \leq u^{(i)}(t)=\int_{0}^{t} u^{(i+1)}(s) d s \leq$ $\max _{0 \leq t \leq 1} u^{(i+1)}(t) \leq\|u\|=\rho, t \in[0,1], i=0,1, \ldots, n-3$. Then by (2.16) we have, for $t \in[0,1]$,

$$
\begin{aligned}
(T u)^{(n-2)}(t) & =\int_{0}^{1} H(t, s) f\left(s, u(s), u^{\prime}(s), \ldots, u^{(n-2)}(s)\right) d s \\
& \leq m \rho \int_{0}^{1} H(t, s) d s \leq \rho=\|u\| .
\end{aligned}
$$

This implies that $\|T u\| \leq\|u\|$ for $u \in \partial K_{\rho}$. By the point (1) in Lemma 1.1, we have $i_{K}\left(T, K_{\rho}\right)=1$.

Lemma 2.7 Assume that $\left(H_{1}\right),\left(H_{2}\right)$ hold and $f$ satisfies

$$
f_{\delta \rho, \rho} \geq \delta M \quad \text { and } \quad u \neq T u \quad \text { for } u \in \partial \Omega_{\rho}
$$

then $i_{K}\left(T, K_{\rho}\right)=0$.

Proof Let $e(t)=\frac{t^{n-2}}{(n-2) !}, t \in[0,1]$, then $e \in K$ with $\|e\|=1$. Next we prove that

$$
u \neq T u+\lambda e, \quad u \in \partial \Omega_{\rho}, \lambda>0 .
$$

In fact, if not, then there exist $u_{0} \in \partial \Omega_{\rho}$ and $\lambda_{0}>0$ such that $u_{0}=T u_{0}+\lambda_{0} e$. By (2.17) and the point (d) in Lemma 2.5, we have, for $t \in[\delta, 1-\delta]$,

$$
\begin{aligned}
u_{0}^{(n-2)}(t) & =\left(T u_{0}\right)^{(n-2)}(t)+\lambda_{0} e^{(n-2)}(t) \\
& =\int_{0}^{1} H(t, s) f\left(s, u_{0}(s), u_{0}^{\prime}(s), \ldots, u_{0}^{(n-2)}(s)\right) d s+\lambda_{0} \\
& \geq \int_{\delta}^{1-\delta} H(t, s) f\left(s, u_{0}(s), u_{0}^{\prime}(s), \ldots, u_{0}^{(n-2)}(s)\right) d s+\lambda_{0} \\
& \geq \delta M \rho \int_{\delta}^{1-\delta} H(t, s) d s+\lambda_{0} \geq \delta \rho+\lambda_{0}>\delta \rho .
\end{aligned}
$$

This implies that $\min _{t \in[\delta, 1-\delta]} u_{0}^{(n-2)}(t)>\delta \rho$, and so by the point (c) in Lemma 2.5 , this is a contradiction. It follows from the point (2) of Lemma 1.1 that $i_{K}\left(T, K_{\rho}\right)=0$.

\section{Main results}

In the following, we shall give the main results on the existence of multiple positive solutions of BVP (1.1) and (1.2).

Theorem 3.1 Suppose that $\left(H_{1}\right)$ and $\left(H_{2}\right)$ are satisfied. In addition, assume that one of the following conditions holds. 
$\left(H_{3}\right)$ There exist $\rho_{1}, \rho_{2}, \rho_{3} \in(0,+\infty)$ with $\rho_{1}<\delta \rho_{2}$ and $\rho_{2}<\rho_{3}$ such that

$$
f^{0, \rho_{1}} \leq m, \quad f_{\delta \rho_{2}, \rho_{2}} \geq \delta M, \quad u \neq T u \quad \text { for } u \in \partial \Omega_{\rho_{2}} \quad \text { and } f^{0, \rho_{3}} \leq m .
$$

$\left(H_{4}\right)$ There exist $\rho_{1}, \rho_{2}, \rho_{3} \in(0,+\infty)$ with $\rho_{1}<\rho_{2}<\delta \rho_{3}$ such that

$$
f_{\delta \rho_{1}, \rho_{1}} \geq \delta M, \quad f^{0, \rho_{2}} \leq m, \quad u \neq T u \quad \text { for } u \in \partial K_{\rho_{2}} \text { and } f_{\delta \rho_{3}, \rho_{3}} \geq \delta M .
$$

Then BVP (1.1) and (1.2) has two nondecreasing positive solutions $u_{1}, u_{2}$ in K. Moreover, if in $\left(H_{3}\right) f^{0, \rho_{1}} \leq m$ is replaced by $f^{0, \rho_{1}}<m$, then $B V P(1.1)$ and (1.2) has a third nondecreasing positive solution $u_{3} \in K_{\rho_{1}}$.

Proof Assume that $\left(H_{3}\right)$ holds. We show that either $T$ has a fixed point $u_{1} \in \partial K_{\rho_{1}}$ or in $\Omega_{\rho_{2}} \backslash \bar{K}_{\rho_{1}}$. If $u \neq T u$ for $u \in \partial K_{\rho_{1}} \cup \partial K_{\rho_{3}}$, by Lemmas 2.6 and 2.7, we have $i_{K}\left(T, K_{\rho_{1}}\right)=1$, $i_{K}\left(T, \Omega_{\rho_{2}}\right)=0$, and $i_{K}\left(T, K_{\rho_{3}}\right)=1$. By Lemma $2.5(\mathrm{~b})$, we have $\bar{K}_{\rho_{1}} \subset K_{\delta \rho_{2}} \subset \Omega_{\rho_{2}}$ since $\rho_{1}<\delta \rho_{2}$. It follows from Lemma 1.1(3) that $T$ has a fixed point $u_{1} \in \Omega_{\rho_{2}} \backslash \bar{K}_{\rho_{1}}$. Similarly, $T$ has a fixed point $u_{2} \in K_{\rho_{3}} \backslash \bar{\Omega}_{\rho_{2}}$. The proof is similar when $\left(H_{4}\right)$ holds.

Corollary 3.1 If there exists $\rho>0$ such that one of the following conditions holds:

$\left(H_{5}\right) \quad 0 \leq f^{0}<m, f_{\delta \rho, \rho} \geq \delta M, u \neq$ Tu for $u \in \partial \Omega_{\rho}, 0 \leq f^{\infty}<\frac{m}{n-1}$,

$\left(H_{6}\right) \quad M<f_{0} \leq \infty, f^{0, \rho} \leq m, u \neq$ Tu for $u \in \partial K_{\rho}, M<f_{\infty} \leq \infty$,

then BVP (1.1) and (1.2) has at least two nondecreasing positive solutions in $K$.

Proof We show that $\left(H_{5}\right)$ implies $\left(H_{3}\right)$. It is easy to verify that $0 \leq f^{0}<m$ implies that there exists $\rho_{1} \in(0, \delta \rho)$ such that $f^{0, \rho_{1}}<m$. Let $k \in\left(f^{\infty}, \frac{m}{n-1}\right)$, by $f^{\infty}<\frac{m}{n-1}$, there exists $r>\rho$ such that $f\left(t, u_{0}, u_{1}, \ldots, u_{n-2}\right) \leq k\left(u_{0}+u_{1}+\cdots+u_{n-2}\right)$ for $t \in[0,1], u_{0}+u_{1}+\cdots+u_{n-2} \in[r,+\infty)$.

Let

$$
\begin{aligned}
& M^{\prime}=\max \left\{\max _{t \in[0,1]} f\left(t, u_{0}, u_{1}, \ldots, u_{n-2}\right): u_{0}+u_{1}+\cdots+u_{n-2} \in[0, r]\right\}, \\
& \rho_{3}>\max \left\{\frac{M^{\prime}}{m-(n-1) k}, r\right\},
\end{aligned}
$$

then for $\left(u_{0}, u_{1}, \ldots, u_{n-2}\right) \in\left[0, \rho_{3}\right]^{n-1}$, we have

$$
\max _{t \in[0,1]} f\left(t, u_{0}, u_{1}, \ldots, u_{n-2}\right) \leq M^{\prime}+k\left(u_{0}+u_{1}+\cdots+u_{n-2}\right) \leq M^{\prime}+k(n-1) \rho_{3}<m \rho_{3} .
$$

This implies that $f^{0, \rho_{3}} \leq m$ and $\left(H_{3}\right)$ holds. Similarly, $\left(H_{6}\right)$ implies $\left(H_{4}\right)$. This completes the proof.

Remark 3.1 We establish the multiplicity of monotone positive solutions for a higher order differential equation with integral boundary conditions, and we allow the nonlinearity $f$ to contain derivatives of the unknown function $u(t)$ up to $n-2$ order, so our work improves and generalizes the results of [2] to some degree. 


\section{Competing interests}

The authors declare that they have no competing interests.

\section{Authors' contributions}

$\mathrm{XH}$ wrote the first manuscript and $\mathrm{LL}$ corrected and improved the final version. Both authors read and approved the final manuscript.

\section{Acknowledgements}

Research supported by the National Natural Science Foundation of China (11371221, 11201260), the Specialized Research Fund for the Doctoral Program of Higher Education of China (20123705120004, 20123705110001), a Project of Shandong Province Higher Educational Science and Technology Program (J11LA06) and Foundation of Qufu Normal University (BSQD20100103)

\section{Received: 17 January 2014 Accepted: 19 March 2014 Published: 28 Mar 2014}

\section{References}

1. Eloe, PW, Ahmad, B: Positive solutions of a nonlinear $n$th order boundary value problem with nonlocal conditions. Appl. Math. Lett. 18, 521-527 (2005)

2. Graef, JR, Yang, B: Positive solutions to a multi-point higher order boundary value problem. J. Math. Anal. Appl. 316, 409-421 (2006)

3. Graef, JR, Henderson, J, Wong, PJY, Yang, B: Three solutions of an $n$th order three-point focal type boundary value problem. Nonlinear Anal. 69, 3386-3404 (2008)

4. Hao, X, Liu, L, Wu, Y: Positive solutions for second order differential systems with nonlocal conditions. Fixed Point Theory 13, 507-516 (2012)

5. Hao, X, Liu, L, Wu, Y: On positive solutions of m-point nonhomogeneous singular boundary value problem. Nonlinear Anal. 73, 2532-2540 (2010)

6. Henderson, J, Luca, R: On a system of second-order multi-point boundary value problems. Appl. Math. Lett. 25, 2089-2094 (2012)

7. Karaca, IY: Positive solutions of an $n$th order multi-point boundary value problem. J. Comput. Anal. Appl. 14, 181-193 (2012)

8. Ma, R: Existence of positive solutions for superlinear semipositone $m$-point boundary-value problems. Proc. Edinb. Math. Soc 46, 279-292 (2003)

9. Hao, $X, X u, N$, Liu, L: Existence and uniqueness of positive solutions for fourth-order $m$-point boundary value problems with two parameters. Rocky Mt. J. Math. 43, 1161-1180 (2013)

10. Zhang, X: Eigenvalue of higher-order semipositone multi-point boundary value problems with derivatives. Appl. Math. Comput. 201, 361-370 (2008)

11. Zhang, X, Liu, L: A necessary and sufficient condition of positive solutions for nonlinear singular differential systems with four-point boundary conditions. Appl. Math. Comput. 215, 3501-3508 (2010)

12. Zhang, $X$, Liu, L: Positive solutions of four-order multi-point boundary value problems with bending term. Appl. Math. Comput. 194, 321-332 (2007)

13. Gallardo, JM: Second order differential operators with integral boundary conditions and generation of semigroups. Rocky Mt. J. Math. 30, 1265-1292 (2000)

14. Karakostas, GL, Tsamatos, PC: Multiple positive solutions of some Fredholm integral equations arisen from nonlocal boundary-value problems. Electron. J. Differ. Equ. 2002, 1-17 (2002)

15. Lomtatidze, A, Malaguti, L: On an nonlocal boundary-value problems for second order nonlinear singular differential equations. Georgian Math. J. 7, 133-154 (2000)

16. Boucherif, A: Second-order boundary value problems with integral boundary conditions. Nonlinear Anal. 70, 364-371 (2009)

17. Feng, M, Ji, D, Ge, W: Positive solutions for a class of boundary-value problem with integral boundary conditions in Banach spaces. J. Comput. Appl. Math. 222, 351-363 (2008)

18. Hao, X, Liu, L, Wu, Y, Sun, Q: Positive solutions for nonlinear $n$ th-order singular eigenvalue problem with nonlocal conditions. Nonlinear Anal. 73, 1653-1662 (2010)

19. Hao, $X, L i u, L, W u, Y, X u, N$ : Multiple positive solutions for singular $n$ th-order nonlocal boundary value problem in Banach spaces. Comput. Math. Appl. 61, 1880-1890 (2011)

20. Infante, G, Webb, JRL: Nonlinear non-local boundary-value problems and perturbed Hammerstein integral equations. Proc. Edinb. Math. Soc. 49, 637-656 (2006)

21. Jiang, J, Liu, L, Wu, Y: Second-order nonlinear singular Sturm-Liouville problems with integral boundary conditions. Appl. Math. Comput. 215, 1573-1582 (2009)

22. Kang, P, Wei, Z: Three positive solutions of singular nonlocal boundary value problems for systems of nonlinear second-order ordinary differential equations. Nonlinear Anal. 70, 444-451 (2008)

23. Kong, L: Second order singular boundary value problems with integral boundary conditions. Nonlinear Anal. 72 , 2628-2638 (2010)

24. $\mathrm{Ma}, \mathrm{H}$ : Symmetric positive solutions for nonlocal boundary value problems of fourth order. Nonlinear Anal. 68 645-651 (2008)

25. Webb, JRL: Nonlocal conjugate type boundary value problems of higher order. Nonlinear Anal. 71, 1933-1940 (2009)

26. Webb, JRL, Infante, G: Positive solutions of nonlocal boundary value problems: a unified approach. J. Lond. Math. Soc 74, 673-693 (2006)

27. Webb, JRL, Infante, G: Positive solutions of nonlocal boundary value problems involving integral conditions. Nonlinear Differ. Equ. Appl. 15, 45-67 (2008)

28. Liu, L, Hao, X, Wu, Y: Positive solutions for singular second order differential equations with integral boundary conditions. Math. Comput. Model. 57, 836-847 (2013)

29. Yang, Z: Existence and nonexistence results for positive solutions of an integral boundary value problem. Nonlinear Anal. 65, 1489-1511 (2006) 
30. Yang, Z: Existence of nontrivial solutions for a nonlinear Sturm-Liouville problem with integral boundary conditions. Nonlinear Anal. 68, 216-225 (2008)

31. Zhang, X, Feng, M, Ge, W: Symmetric positive solutions for $p$-Laplacian fourth-order differential equations with integral boundary conditions. J. Comput. Appl. Math. 222, 561-573 (2008)

32. Zhang, $X$, Han, Y: Existence and uniqueness of positive solutions for higher order nonlocal fractional differential equations. Appl. Math. Lett. 25, 555-560 (2012)

33. Lan, KQ: Multiple positive solutions of semilinear differential equations with singularities. J. Lond. Math. Soc. 63 690-704 (2001)

34. Guo, D, Lakshmikantham, V: Nonlinear Problems in Abstract Cones. Academic Press, New York (1988)

10.1186/1687-2770-2014-74

Cite this article as: Hao and Liu: Multiple monotone positive solutions for higher order differential equations with integral boundary conditions. Boundary Value Problems 2014, 2014:74

\section{Submit your manuscript to a SpringerOpen ${ }^{\circ}$ journal and benefit from:}

- Convenient online submission

- Rigorous peer review

- Immediate publication on acceptance

- Open access: articles freely available online

- High visibility within the field

- Retaining the copyright to your article 